\title{
Jet substructure measurements in heavy-ion collisions with ALICE
}

\author{
James Mulligan $^{a, b}$ for the ALICE Collaboration \\ ${ }^{a}$ Nuclear Science Division, Lawrence Berkeley National Laboratory, Berkeley, California 94720, USA \\ ${ }^{b}$ Physics Department, University of California, Berkeley, CA 94720, USA \\ E-mail: james.mulligan@berkeley.edu
}

Jet substructure, defined by observables constructed from the distribution of constituents within a jet, provides the versatility to tailor observables to specific regions of QCD radiation phase space. This flexibility provides exciting new opportunities to study jet quenching in heavy-ion collisions and to ultimately help reveal the nature of the quark-gluon plasma. The ALICE detector is particularly well-suited to jet substructure measurements in heavy-ion collisions due to its high-precision tracking system. In these proceedings, we report several new jet substructure measurements in $\mathrm{Pb}-\mathrm{Pb}$ collisions at $\sqrt{s_{\mathrm{NN}}}=5.02 \mathrm{TeV}$ with ALICE. These include the first fully corrected measurements of the groomed jet momentum splitting fraction, $z_{\mathrm{g}}$, and the groomed jet radius, $\theta_{\mathrm{g}} \equiv R_{\mathrm{g}} / R$, as well as $N$-subjettiness and the fragmentation distribution of reclustered subjets. These measurements are compared to theoretical calculations and provide new constraints on the physics underlying jet quenching.

\footnotetext{
*** The European Physical Society Conference on High Energy Physics (EPS-HEP2021), *** *** 26-30 July $2021 * * *$

*** Online conference, jointly organized by Universität Hamburg and the research center DESY ***
} 


\section{Introduction}

At the Large Hadron Collider (LHC) and Relativistic Heavy Ion Collider (RHIC), high-energy nuclear collisions produce hot, dense droplets of a phase of matter known as the quark-gluon plasma (QGP), consisting of deconfined quarks and gluons [1]. While the interactions between the quarks and gluons in the QGP are too weak to bind them into nucleons, they remain sufficiently strong to form a strongly coupled liquid. The microscopic structure of this liquid remains unknown, and it is not clear whether quasiparticle degrees of freedom emerge at some scale. Understanding the fundamental degrees of freedom of this strongly coupled system and how they arise from a quantum field theory is one of the major outstanding questions in quantum chromodynamics (QCD).

In order to investigate the microscopic structure of the QGP, one needs to probe it at a variety of length scales. Jets are well suited for this task, since not only can their transverse momentum span a wide range of values but also the internal pattern of particles within jets, known as jet substructure, enables the design of observables to target specific regions of QCD phase space [2]. The study of jet modification in heavy-ion collisions compared to proton-proton collisions, known as jet quenching, has established that jet-medium interactions result in a suppression of jet yields and an excess of soft, wide angle radiation [3-5]. There remain many unknowns in jet quenching theory, however, including the roles of color coherence, medium response, and the space-time picture of the parton shower. Measurements of suitably chosen jet substructure observables aim to test these aspects of jet quenching models, and ultimately provide a path to determine the microscopic nature of the QGP using global fits (see e.g. Ref. [6]).

In these proceedings, we highlight a selection of recent jet substructure results from the ALICE experiment [7]. While ALICE has performed a variety of measurements in proton-proton collisions, which explore the transition from the perturbative to the nonperturbative regimes (see e.g. Ref. [8]), here we focus on measurements in heavy-ion collisions. We emphasize observables that are directly comparable to theoretical models, meaning that they are (i) analytically calculable in pQCD, and (ii) corrected for detector effects and underlying event fluctuations. All results presented utilize jets reconstructed from charged particles at midrapidity using the anti- $k_{\mathrm{T}}$ algorithm [9].

\section{Groomed jet observables: $z_{\mathrm{g}}$ and $\theta_{\mathrm{g}}$}

Jet grooming techniques have been applied to heavy-ion collisions to explore whether jet quenching modifies the hard substructure of jets [10-20]. The Soft Drop grooming algorithm identifies a single splitting within a jet from a grooming condition $z>z_{\text {cut }} \theta^{\beta}$, where $z$ is the fraction of transverse momentum carried by the sub-leading prong, $\theta$ is the relative angular distance between the leading and sub-leading prong, and $\beta$ and $z_{\text {cut }}$ are tunable parameters [21-23]. The groomed splitting is then characterized by two kinematic observables: the groomed momentum fraction, $z_{\mathrm{g}}$ [24], and the (scaled) groomed jet radius, $\theta_{\mathrm{g}}$ [25]. By using strong grooming conditions [26], ALICE measured the $z_{\mathrm{g}}$ and $\theta_{\mathrm{g}}$ distributions in heavy-ion collisions. Figure 1 (left) shows that the $z_{\mathrm{g}}$ distribution is not significantly modified in heavy-ion collisions, suggesting that medium-induced radiations are not sufficiently hard to pass the grooming condition. On the other hand, Fig. 1 (right) shows a narrowing of the $\theta_{\mathrm{g}}$ distributions in $\mathrm{Pb}-\mathrm{Pb}$ collisions relative to $\mathrm{pp}$ collisions [27]. These measurements are compared to a variety of jet quenching models [10, 12, 14-16, 28-33], 

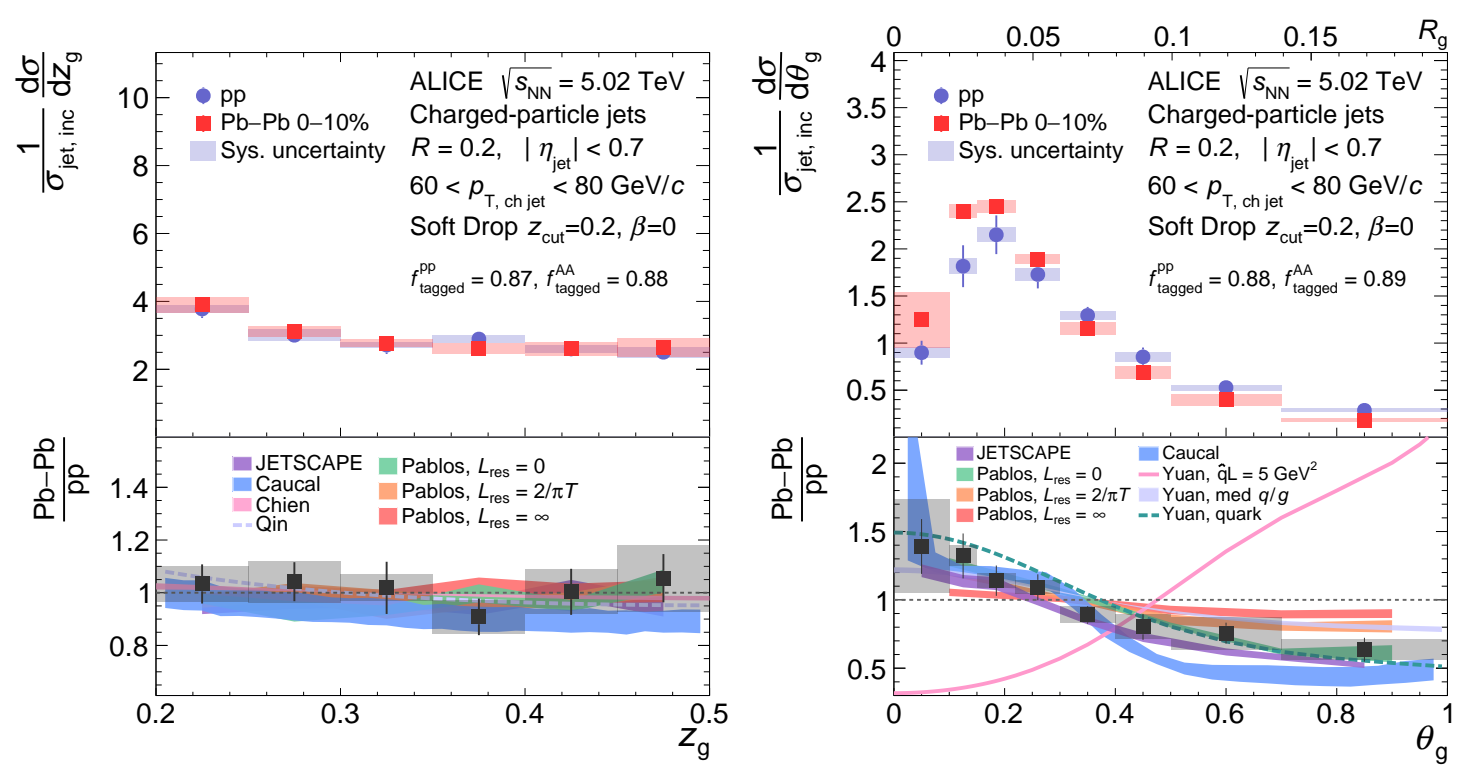

Figure 1: Left: Measurements of $z_{\mathrm{g}}$ (left) and $\theta_{\mathrm{g}}$ (right) in $\mathrm{Pb}-\mathrm{Pb}$ compared to pp collisions, along with comparisons to several theoretical models [27].

most of which capture the qualitative narrowing effect observed. This behavior is consistent with models implementing an incoherent interaction of the jet shower constituents with the medium, but is also consistent with medium-modified quark/gluon fractions with fully coherent energy loss presenting the opportunity for future measurements to disentangle them definitively.

\section{Sub-jet fragmentation}

In heavy-ion collisions, measurements of reclustered sub-jets have been proposed as sensitive probes of jet quenching [34-36]. We first inclusively reconstruct charged-particle jets with the anti$k_{\mathrm{T}}$ jet algorithm and jet radius $R$, and then recluster the charged jet constituents with the anti- $k_{\mathrm{T}}$ jet algorithm and sub-jet radius $r<R$. We consider the fraction of transverse momentum carried by the sub-jet compared to the initial jet: $z_{r}=p_{\mathrm{T}}^{\text {ch subjet }} / p_{\mathrm{T}}^{\text {ch jet }}$. Figure 2 (left) shows the distribution of leading sub-jets with $r=0.1$ for $R=0.4$ jets in both $\mathrm{pp}$ and $\mathrm{Pb}-\mathrm{Pb}$ collisions. The distributions are compared to theoretical predictions [28-30,34,37] that accurately reproduce a mild rising trend of the ratio with $z_{r}$, which can be attributed to jet collimation. The ratio then falls as $z_{r} \rightarrow 1$, which may be due to the large quark/gluon fraction at $z_{r} \rightarrow 1$. These measurements offer an opportunity to probe higher $z$ than hadron fragmentation measurements, and are an important ingredient for future tests of the universality of in-medium jet fragmentation functions.

\section{N-subjettiness}

Semi-inclusive hadron-jet correlations are well-suited to statistical background subtraction procedures in heavy-ion collisions, which allows jet measurements at low $p_{\mathrm{T}}$ and large $R[38,39]$. Recently, ALICE measured the N-subjettiness $\tau_{\mathrm{N}}[40,41]$ of jets recoiling from a high- $p_{\mathrm{T}}$ hadron 

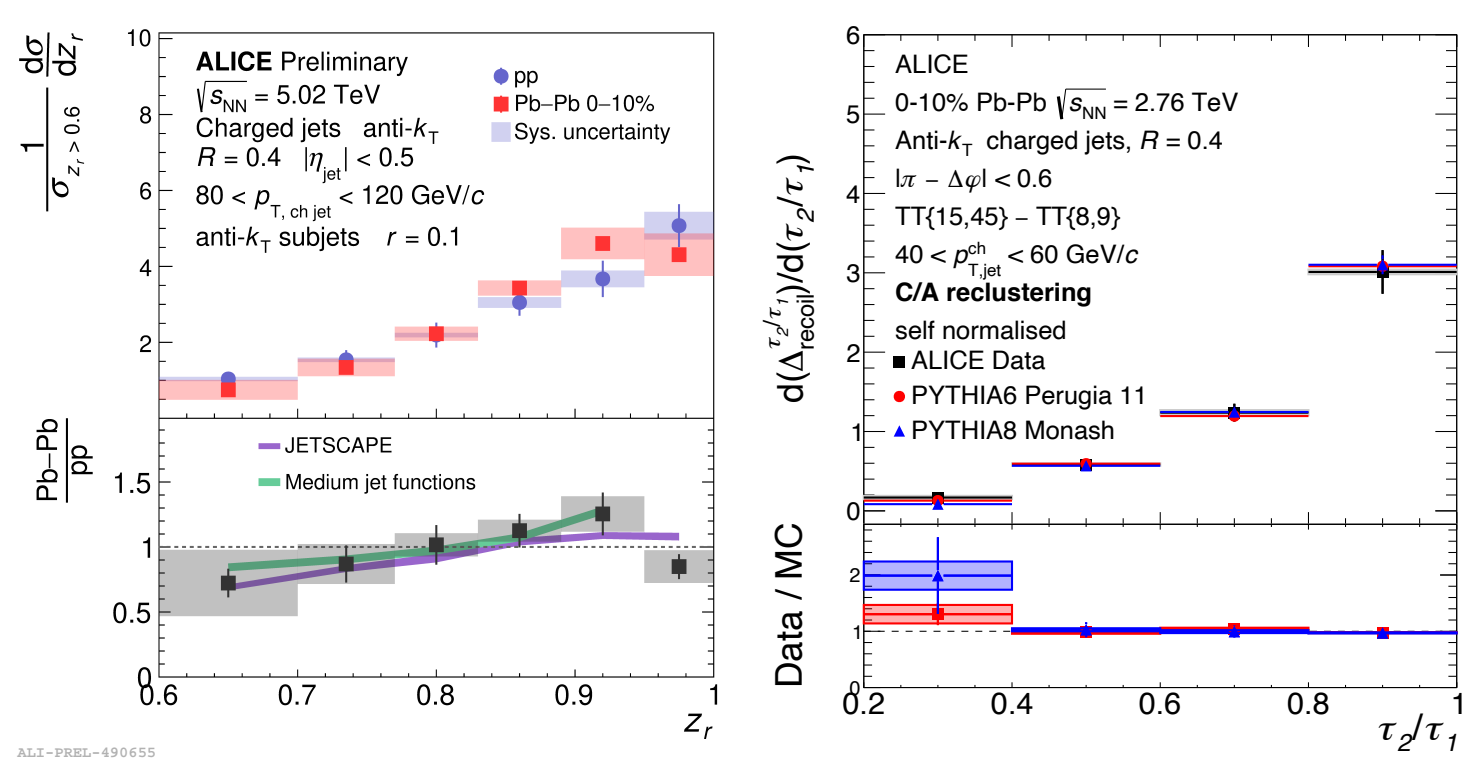

Figure 2: Left: ALICE measurements of leading sub-jet fragmentation in $\mathrm{pp}$ and $\mathrm{Pb}-\mathrm{Pb}$ collisions, compared to theoretical predictions [28-30, 34, 37]. Right: Measurements of the N-subjettiness ratio distribution $\tau_{2} / \tau_{1}$ in $\mathrm{Pb}-\mathrm{Pb}$ collisions [42] compared to PYTHIA [43].

[42]. Figure 2 (right) shows the distribution of per-trigger semi-inclusive yields of the $\tau_{2} / \tau_{1}$ ratio in $\mathrm{Pb}-\mathrm{Pb}$ collisions compared to PYTHIA [43]. There is no significant modification observed in the pronginess of jets in heavy-ion collisions. This suggests that medium-induced emissions are not sufficiently hard to produce a distinct secondary prong, in line with the lack of modification of the observed $z_{\mathrm{g}}$ distributions [27].

\section{Conclusion}

We have presented several new ALICE measurements of jet substructure in heavy-ion collisions, which are producing an emerging picture of jet quenching phenomenology: hard splittings are not strongly modified, as evidenced by $z_{\mathrm{g}}$ and $\tau_{N}$, but there is a strong collimation or filtering effect of wide jets, as evidenced by $\theta_{\mathrm{g}}$. The medium-induced soft splitting responsible for this filtering may be exposed in regions dominated by quark jets, as suggested by high- $z_{r}$ sub-jet fragmentation. Together, these observables present opportunities for future high statistics and/or multi-differential measurements in LHC Run 3 to achieve increasingly precise constraints on jet quenching models, and offer prospects to constrain physical properties of the QGP using global analyses.

\section{References}

[1] W. Busza, K. Rajagopal and W. van der Schee, ARNPS 68 (2018), 339.

[2] A. J. Larkoski, I. Moult and B. Nachman, Phys. Rep. 841 (2020), 1-63.

[3] G.-Y. Qin and X.-N. Wang, IJMPE 24 (2015), 1530014.

[4] J.-P. Blaizot and Y. Mehtar-Tani, IJMPE 24 (2015), 1530012.

[5] A. Majumder and M. van Leeuwen, PPNP 66 (2011), 41 . 
[6] JETSCAPE collaboration, S. Cao et al., PRC 104 (2021), 024905.

[7] ALICE Collaboration, JINST 3 (2008), S08002.

[8] ALICE collaboration, S. Acharya et al., 2107.11303.

[9] M. Cacciari, G. P. Salam and G. Soyez, JHEP 04 (2008), 063.

[10] Y.-T. Chien and I. Vitev, PRL 119 (2017), 112301.

[11] Y. Mehtar-Tani and K. Tywoniuk, JHEP 2017 (2017), 125.

[12] N.-B. Chang, S. Cao and G.-Y. Qin, PLB 781 (2018), 423-432.

[13] R. Kunnawalkam Elayavalli and K. C. Zapp, JHEP 7 (2017), 141.

[14] P. Caucal, E. Iancu and G. Soyez, JHEP 10 (2019), 273.

[15] F. Ringer, B.-W. Xiao and F. Yuan, PLB 808 (2020), 135634.

[16] J. Casalderrey-Solana, G. Milhano, D. Pablos and K. Rajagopal, JHEP 01 (2020), 044.

[17] H. A. Andrews and et al., JPG 47 (2020), 065102.

[18] CMS Collaboration, PRL 120 (2018), 142302.

[19] ALICE Collaboration, PLB 802 (2020), 135227.

[20] CMS Collaboration, JHEP 2018 (2018), 161.

[21] A. J. Larkoski, S. Marzani, G. Soyez and J. Thaler, JHEP 05 (2014), 146.

[22] M. Dasgupta, A. Fregoso, S. Marzani and G. P. Salam, JHEP 09 (2013), 029.

[23] A. J. Larkoski, S. Marzani and J. Thaler, PRD 91 (2015), 111501.

[24] P. Cal, K. Lee, F. Ringer and W. J. Waalewijn, 2106. 04589.

[25] Z.-B. Kang, K. Lee, X. Liu, D. Neill and F. Ringer, JHEP 2020 (2020), 54.

[26] J. Mulligan and M. Ploskon, PRC 102 (2020), 044913.

[27] ALICE collaboration, S. Acharya et al., 2107.12984.

[28] J. H. Putschke et al., 1903.07706.

[29] Y. He, T. Luo, X.-N. Wang and Y. Zhu, PRC 91 (2015), 054908.

[30] A. Majumder, PRC 88 (2013), 014909.

[31] P. Caucal, E. Iancu, A. Mueller and G. Soyez, PRL 120 (2018), 232001.

[32] J. Casalderrey-Solana, D. C. Gulhan, J. G. Milhano, D. Pablos and K. Rajagopal, JHEP 019 (2014), 019.

[33] Z. Hulcher, D. Pablos and K. Rajagopal, JHEP 010 (2018), 10.

[34] Z.-B. Kang, F. Ringer and W. J. Waalewijn, JHEP 07 (2017), 064.

[35] D. Neill, F. Ringer and N. Sato, JHEP 07 (2021), 041.

[36] L. Apolinário, J. G. Milhano, M. Ploskon and X. Zhang, EPJC 78 (2018), 529 [1710.07607].

[37] J.-W. Qiu, F. Ringer, N. Sato and P. Zurita, PRL 122 (2019), 252301.

[38] ALICE Collaboration, JHEP 2015 (2015), 170.

[39] STAR Collaboration, PRC 96 (2017), 024905.

[40] I. W. Stewart, F. J. Tackmann and W. J. Waalewijn, PRL 105 (2010), 092002.

[41] J. Thaler and K. Van Tilburg, JHEP 03 (2011), 015.

[42] ALICE collaboration, S. Acharya et al., 2105.04936.

[43] T. Sjostrand et al., CPC 191 (2015), 159. 